Corrigendum

\title{
Corrigendum to "Special Staining of the Liquid-Based Cytopathology Test in Bronchoalveolar Lavage Fluid for Diagnosis of Invasive Pulmonary Aspergillosis with Nonneutropenic Patients"
}

\author{
Yue Hu, ${ }^{1}$ Lin Zheng, ${ }^{2}$ Deng Pan, ${ }^{3}$ Lei Shao, ${ }^{3}$ Xianfa Xu, ${ }^{3}$ Yiming Yu, ${ }^{1}$ Qidong Zhuang $\mathbb{D}^{1}$ \\ Zhongbo Chen $\mathbb{D}^{1},{ }^{1}$ and Zaichun Deng $\mathbb{D}^{1}$ \\ ${ }^{1}$ Department of Pulmonary and Critical Care Medicine, The Affiliated Hospital of Medical School of Ningbo University, \\ 247 Renmin Road, Ningbo, Zhejiang 315020, China \\ ${ }^{2}$ Department of Microbiology, The Affiliated Hospital of Medical School of Ningbo University, 247 Renmin Road, Ningbo, \\ Zhejiang 315020, China \\ ${ }^{3}$ Department of Cell, Clinicopathological Diagnosis Center of Ningbo, 685 Huancheng North Road, Ningbo, \\ Zhejiang 315211, China
}

Correspondence should be addressed to Zhongbo Chen; leonczb@yahoo.com and Zaichun Deng; dengzaichun1964@sina.com Received 27 October 2020; Accepted 27 October 2020; Published 9 November 2020

Copyright $(2020$ Yue Hu et al. This is an open access article distributed under the Creative Commons Attribution License, which permits unrestricted use, distribution, and reproduction in any medium, provided the original work is properly cited.

In the article titled "Special Staining of the Liquid-Based Cytopathology Test in Bronchoalveolar Lavage Fluid for Diagnosis of Invasive Pulmonary Aspergillosis with Nonneutropenic Patients" [1], the author order in the author list was incorrect, where

Yue Hu, Lin Zheng, Deng Pan, Lei Shao, Xianfa Xu, Yiming Yu, Qidong Zhuang, Zaichun Deng, and Zhongbo Chen should have read:

Yue Hu, Lin Zheng, Deng Pan, Lei Shao, Xianfa Xu, Yiming $\mathrm{Yu}$, Qidong Zhuang, Zhongbo Chen, and Zaichun Deng

The correct author order is also shown above in the author information.

\section{References}

[1] Y. Hu, L. Zheng, P. Deng et al., "Special staining of the liquidbased cytopathology test in bronchoalveolar lavage fluid for diagnosis of invasive pulmonary aspergillosis with nonneutropenic patients," Canadian Respiratory Journal, vol. 2020, Article ID 8243473, 9 pages, 2020. 